\title{
PHYSICAL EXAMINATION IS SUFFICIENT FOR THE DIAGNOSIS OF SPRAINED ANKLES
}

\author{
C. N. van DIJK, L. S. L. LIM, P. M. M. BOSSUYT, R. K. MARTI \\ From the University of Amsterdam, The Netherlands
}

W e studied the merits of physical examination after inversion injury of the ankle in $\mathbf{1 6 0}$ consecutive patients. They had an explanatory operation if they had a positive arthrogram and/or positive signs on a delayed physical examination.

To determine the interobserver variation in delayed physical examination, five different examiners were asked to give independent assessment of the injury. Those with limited clinical experience produced more accurate results when physical examination was performed at five days after the injury, rather than within 48 hours.

The specificity and sensitivity of delayed physical examination for the presence or absence of a lesion of an ankle ligament were found to be $84 \%$ and $96 \%$, respectively. The interobserver agreement for the delayed physical examination of the ankle was good (kappa values 0.5, 0.6, 0.6 and 1.0).

Delayed physical examination gives information of diagnostic quality which is equal to that of arthrography, and causes little discomfort to the patient.

J Bone Joint Surg [Br] 1996;78-B:958-62.

Received 12 September 1995; Accepted after revision 20 May 1996

C. N. van Dijk, MD, PhD

L. S. L. Lim, MD

R. K. Marti, MD, PhD

Department of Orthopaedic Surgery

P. M. M. Bossuyt, MD, PhD

Department of Epidemiology

Academic Medical Centre, Meibergdreef 9, 1105 AZ Amsterdam, The Netherlands.

Correspondence should be sent to Dr C. N. van Dijk.

(C)1996 British Editorial Society of Bone and Joint Surgery 0301-620X/96/61283\$2.00
Because of the unreliability of physical examination, various imaging techniques have been developed for the diagnosis of an inversion injury of the ankle. Initially, this was by stress radiography with treatment by immobilisation in a cast, but was then followed by the use of arthrography and operative treatment. In the 1980s, functional treatment was shown to be equally successful, and became the treatment of choice (Kannus and Renström 1991). Diagnostic arthrography and tenography were considered to be costly and unnecessary and stress radiography was regarded as unreliable.

A review of the literature has found no difference in the results of the treatment of single and multiple ligament ruptures (Kannus and Renström 1991). Diagnosis should therefore differentiate between a sprain and a ligament rupture, be cost-effective and allow similarly cost-effective functional treatment (Brooks, Potter and Rainey 1981). Stiell et al (1994) have shown that physical examination is reliable for the diagnosis of an ankle fracture, and we have therefore sought to enhance its reliability in the detection of ruptures of ankle ligaments. We felt that physical examination, carried out several days after injury, might have better diagnostic quality because of diminished pain and swelling.

\section{PATIENTS AND METHODS}

The Accident and Emergency Department at the Amsterdam Academic Medical Centre is accessible to patients without referral by a general practitioner.

Over a 12-month period, we established a registry of all consecutive patients between 18 and 40 years of age who presented within 48 hours of an acute injury to the lateral ankle ligaments. Those with a history of previous ankle sprain were excluded. The study was part of a randomised, controlled trial of different methods of treatment and was approved by our medical ethical committee. All patients gave their fully informed consent.

According to the databank of the Consumer Safety Institute the patients in our series were similar to those examined for ankle sprains in other Dutch hospitals during the same period and had similar details as regards gender, age, place of accident, activity at the time of the accident and type of injury. There were 116 men and 44 women with an average age of 27.3 years. The left ankle was involved in 74 and the right ankle in 86. 


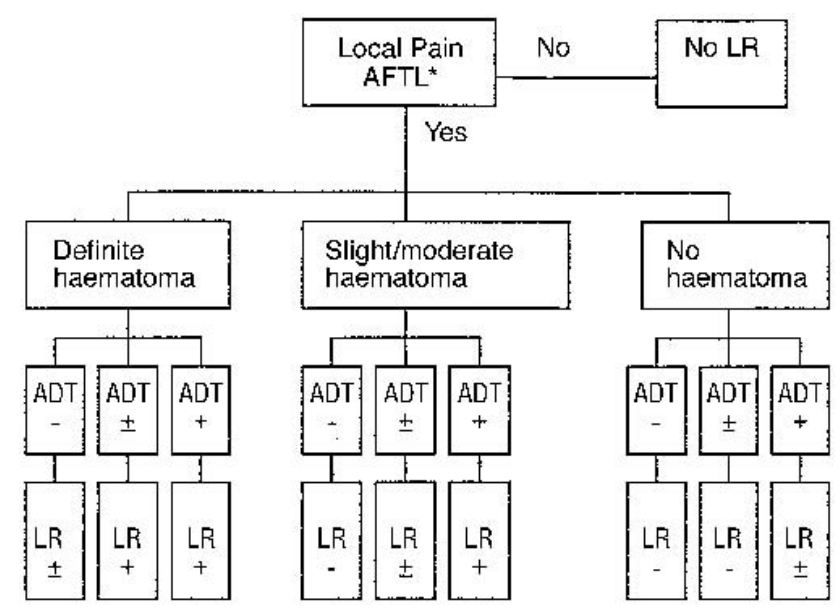

Fig. 1

Assessment of the painful ankle five days after inversion injury (ATFL, anterior talofibular ligament; ADT, anterior-drawer test; LR, ligament rupture)
Within 48 hours of injury we performed physical examination which determined the extent of the swelling, the formation of a haematoma, localisation of pain on palpation and the anterior-drawer test. The evidence of lateral ligament rupture was noted on a standard form as positive, negative or inconclusive. All patients were examined again four to seven days later by an experienced orthopaedic surgeon. In addition, without knowing the result of this examination, they were all independently examined by one of a group of four inexperienced, but well-instructed doctors each of whom assessed the likelihood of a ligament lesion using the same criteria (Figs 1 and 2).

We performed arthrography in each patient but the outcome was neither disclosed to the patient nor the investigator until after the delayed examination.

Patients who were thought to have a lateral ligament rupture at the delayed physical examination or who had a positive arthrogram, were treated by operative suture and then immobilisation in a plaster cast for five days, followed
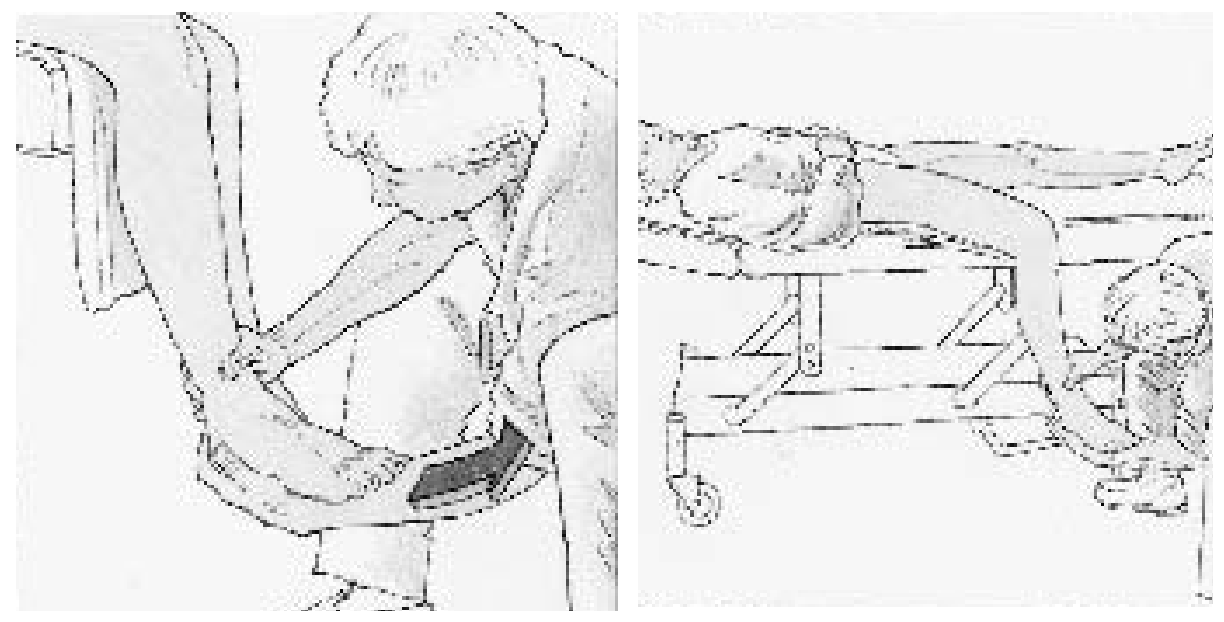

Fig. 2a
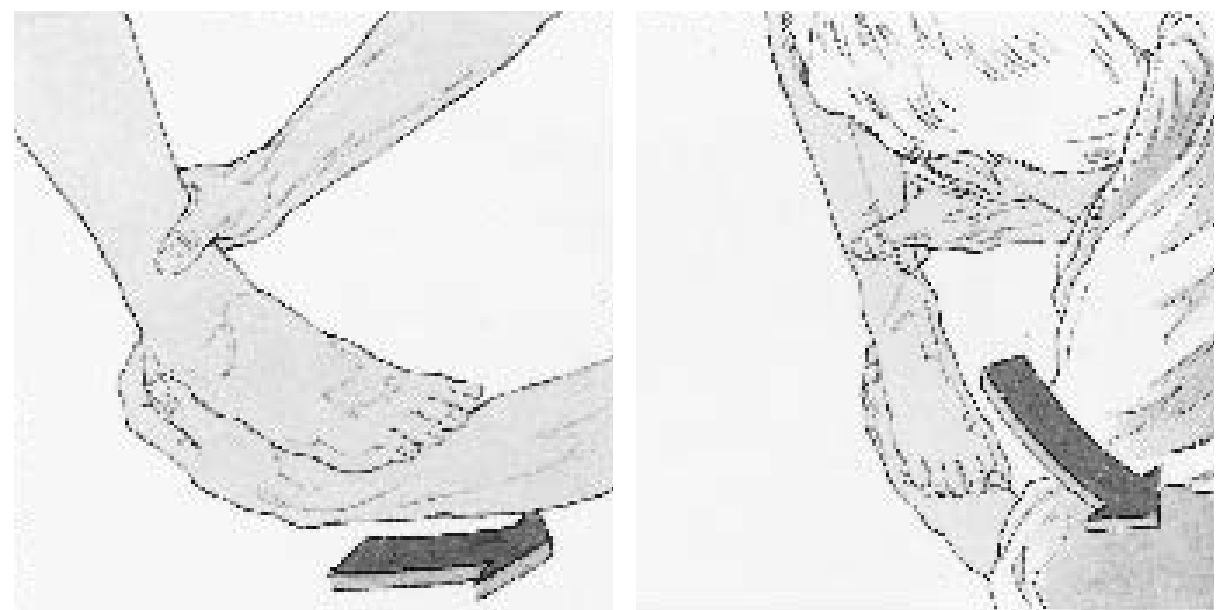

Fig. $2 b$

Anterior-drawer test for right ankle. Figure $2 \mathrm{a}-$ The patient sits at the end of the couch or lies supine with the upper leg supported by the couch and the knee flexed. The ankle is held in $10^{\circ}$ to $15^{\circ}$ of plantar flexion. The examiner's left hand grasps the heel, while the patient's foot rests on the anterior aspect of the examiner's lower arm. Figure $2 \mathrm{~b}-$ The heel is very gently pulled forward. The talus, and with it the foot, rotates anteriorly out of the ankle mortice. The centre of rotation is the intact deltoid ligament. When a rupture of the anterior talofibular ligament is present, 'a dimple' may be seen just in front of the tip of the lateral malleolus. The forward movement of the talus results in a negative pressure which draws the skin inwards at the side of ligament rupture. 

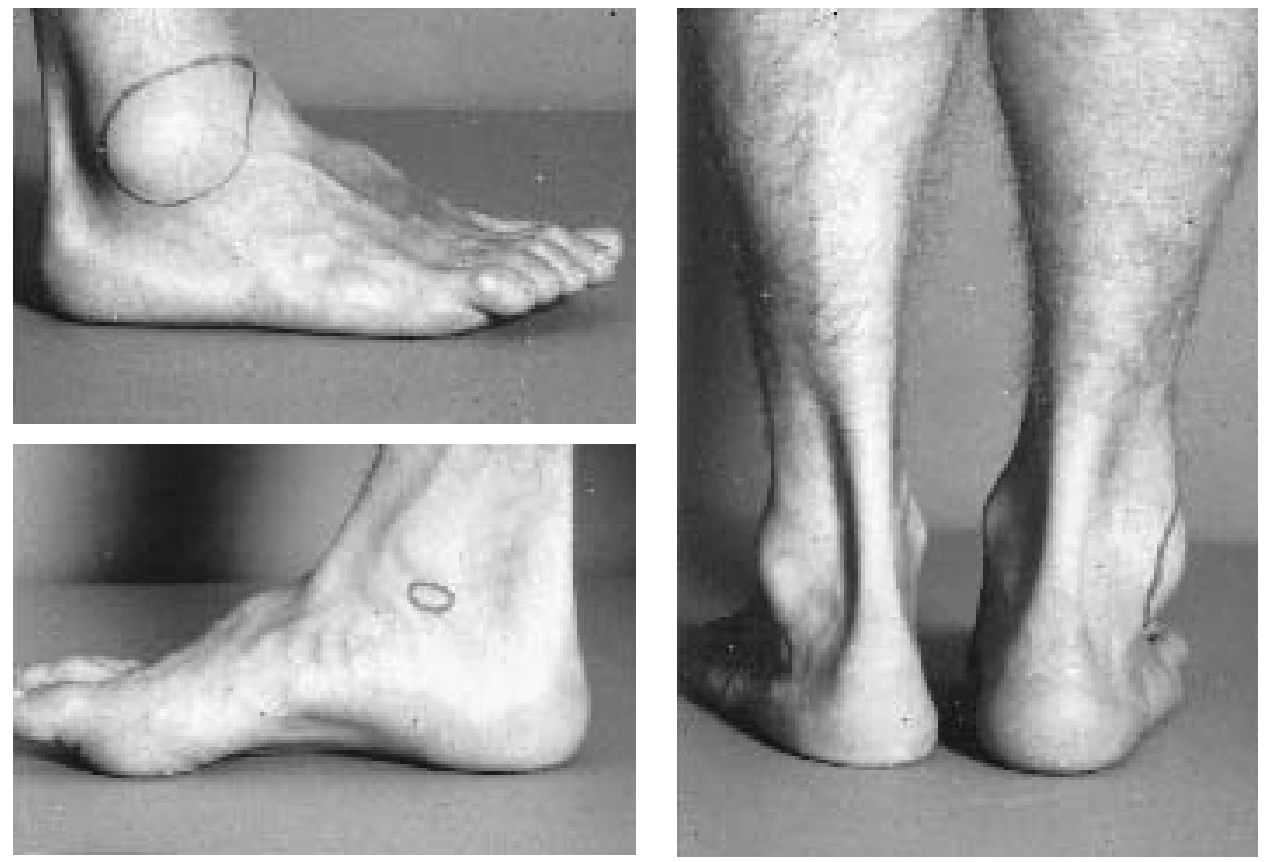

Fig. 3a
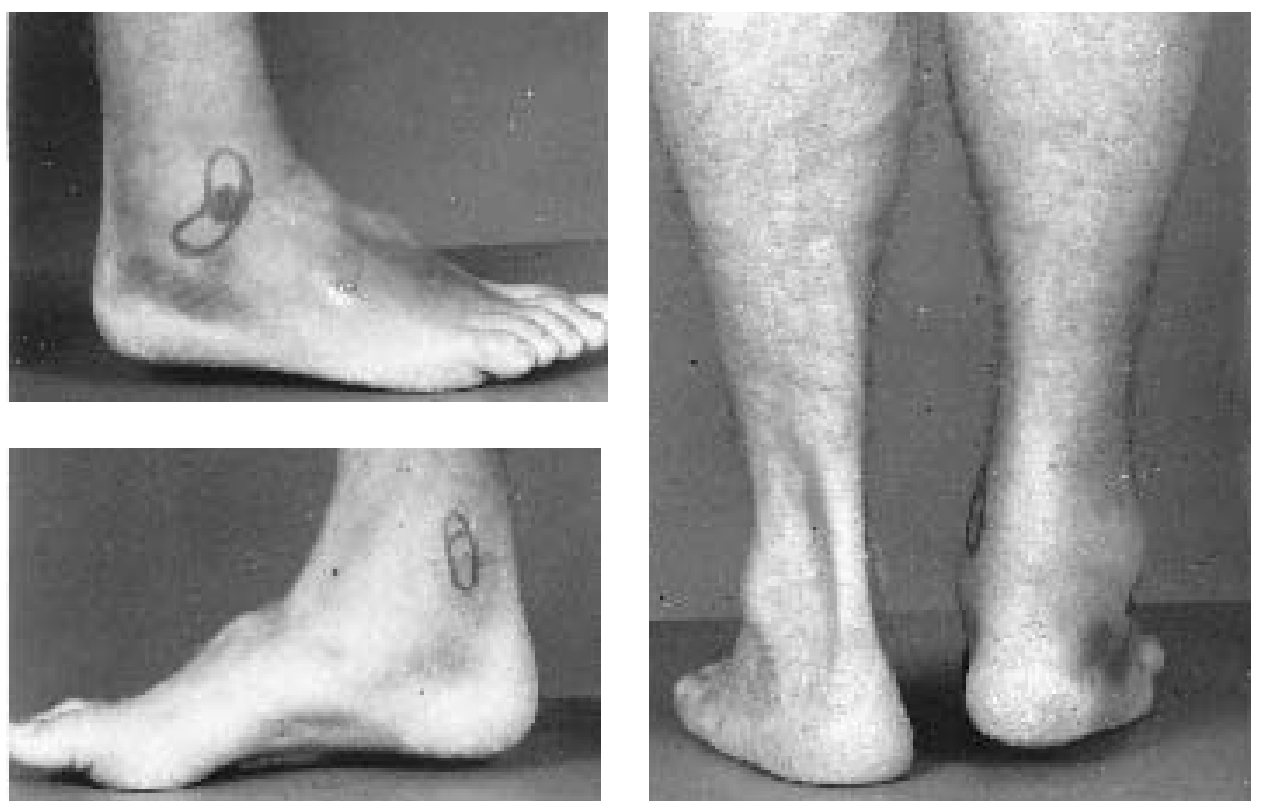

Fig. 3b

A 28-year-old man with inversion injury. The location of pain on palpation is marked on the skin with a felttipped pen. Figure $3 \mathrm{a}$ - Six hours after injury there is localised swelling, diffuse pain, an indecisive anteriordrawer test and pressure pain on the medial side. Figure $3 b-$ Four days after injury there is characteristic discoloration of the skin, acute tenderness over the syndesmosis and the anterior talofibular ligament, a positive anterior-drawer test with a dimple sign, and tenderness with skin discoloration on the medial side. The differences at six hours and at four days are clearly seen.

by functional treatment in a bandage. All patients were followed for at least six months.

Analysis. We studied the specificity, sensitivity and predictive value of the positive and negative results of delayed physical examination. We also compared the result of regarding inconclusive judgements as negative with that of regarding them as positive. To determine interobserver variation in delayed physical examination, we determined kappa values for patients who had been seen by a principal investigator and one of the other examiners. Sections of the 
Table I. Results of physical examination performed five days after trauma compared with arthrography and confirmed by operation

\begin{tabular}{llllll}
\hline & & \multicolumn{2}{c}{ Operative result } & \\
\cline { 3 - 4 } & & Lesion yes & Lesion no & Likelihood ratio \\
\hline Examiner 1 & Ligament lesion & 117 & 6 & 6.07 \\
& No lesion & - & 20 & 0.0 \\
& Indecisive & 5 & 12 & $1 / 7.71$ \\
Arthrography & Ligament lesion & 117 & 11 & 3.31 \\
& No lesion & 4 & 27 & $1 / 21.67$ \\
& Indecisive & 1 & - & 0.0 \\
\hline
\end{tabular}

study were analysed using the Wilcoxon test.

\section{RESULTS}

Twenty-five patients had a negative arthrogram and showed no clinical signs of a ligament lesion. These patients did not have surgery and when they were reviewed after six months the ankle was normal. Of the remaining 135 patients, 122 were found to have a ligament lesion at surgery, which was multiple in 55. Thus, of the 160 patients, 122 had complete rupture of at least one ankle ligament and in the other 38 had intact lateral ligaments.

Physical examination within $\mathbf{4 8}$ hours of injury. Fortysix patients (32 men and 14 women) were evaluated. In many cases it was difficult to apply the method of assessment (Fig. 1). The discoloration of a haematoma was often lacking and it was therefore uncertain whether the swelling was due to a haematoma or oedema. Tenderness was often diffuse, and the drawer test was often unreliable because of pain and swelling. The examiners found that subjective factors often played a role in the assessment of the severity of pain and the ability to bear weight. Pain elicited by performing the drawer test was sometimes, unwittingly, taken as a sign of ligament damage. The sensitivity for this examination was found to be $71 \%$ and its specificity $33 \%$.

Physical examination five days after injury (Fig. 3). Table I gives the results of the physical examination and of arthrography. The prevalence of an ankle ligament lesion in the 160 patients was $76 \%$. When inconclusive judgements were regarded as positive, the overall results were less accurate than when they were regarded as negative. The sensitivity of the delayed physical examination was found to be $96 \%$ and its specificity $84 \%$.

Of the six patients in whom a false-positive physical diagnosis was made, three had a lesion of the syndesmosis, while another had an old rupture of the anterior talofibular ligament with elongation of the healed ligament. Two had only a capsular tear.

Interobserver agreement at five days after injury. The proportion of potential agreement achieved beyond chance was calculated for the patients seen by the principal investigator and also by one of the four other less experienced
Table II. Clinical details and physical findings in patients with and without a lateral ankle ligament lesion, by number and percentage

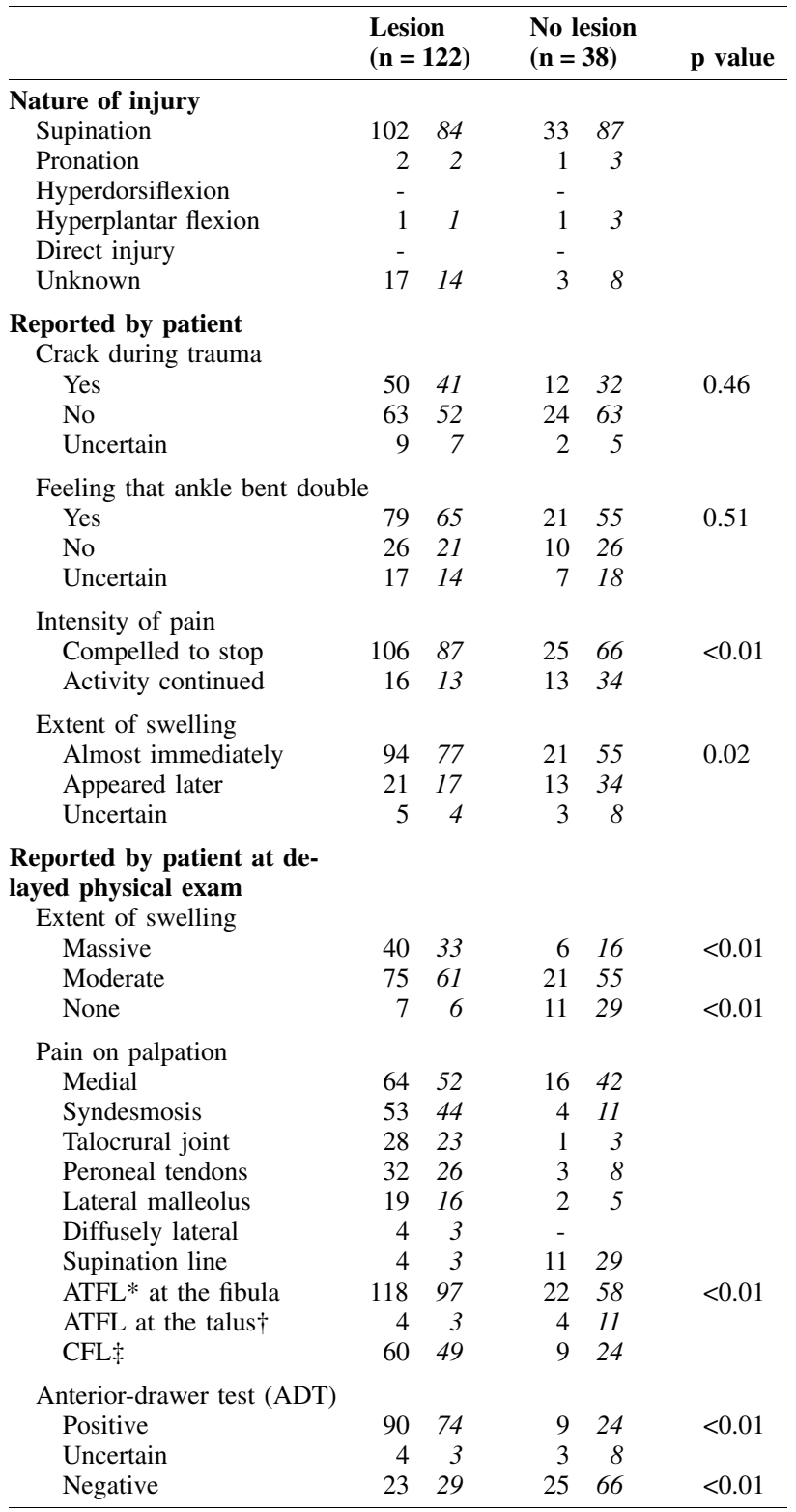

* anterior talofibular ligament

$\dagger$ palpation pain experienced only at this site and not at the level of the fibular attachment

\$Calcaneofibular ligament 
examiners. The kappa values (actual agreement beyond chance divided by the potential agreement beyond chance) were $0.5,1.0,0.6$ and 0.6 respectively.

Analysis of the physical findings (Table II). There was immediate swelling in $78 \%$ of the patients with a ligament lesion compared with $55 \%$ in those without a lesion $(\mathrm{p}=0.02)$. At the time of delayed physical examination moderate swelling was present in most patients from both series.

Absence of swelling was found more often in patients without a ligament lesion, while substantial swelling was seen more often in patients with a lesion $(\mathrm{p}<0.01)$. There was no positive statistical evidence of the value of an audible click or snap at the time of injury $(p=0.46)$. More patients with a ligament lesion had to suspend their normal activities as the result of an injury $(\mathrm{p}<0.01)$.

All patients with a confirmed ligament injury had pain on palpation of the site of the anterior talofibular ligament while in the group without ligament injury, 12 of the 38 patients had no pain on palpation at this site $(\mathrm{p}<0.01)$. In most patients, pain on palpation was not limited to the anterior talofibular ligament. If a positive anterior-drawer test was taken as necessary for diagnosis of a ligament lesion its specificity was $74 \%$ with a sensitivity of $86 \%$ (Table II).

The combination of pain on palpation at the site of the anterior talofibular lesion, lateral discoloration due to a haematoma, and a positive anterior-drawer test gave a $95 \%$ incidence of a definite ligament lesion. When there was no visible discoloration and the anterior-drawer sign was negative an intact lateral ligament was always found. This was also true when no pain could be elicited on palpation of the anterior talofibular ligament.

Only 36 of the 160 patients did not fit into one of these three categories, but most of these (33) were diagnosed correctly. Figure 1 shows the methods by which these diagnoses were reached.

\section{DISCUSSION}

We have compared the diagnostic accuracy of physical examination performed within 48 hours of injury, arthrography, and delayed physical examination. The last showed good interobserver variation and compared favourably with arthrography. We found no significant difference between patients with and without ligament damage with respect to the mechanism of injury, a 'cracking' sound heard at the time of the accident or the patient's story that his ankle had bent double during the injury (Lindstrand 1976a,b; Prins 1978; Nilsson 1982).

Of patients with rupture of an ankle ligament, $87 \%$ had to discontinue their activities compared with $66 \%$ of the patients who did not have a rupture. Nilsson (1982) reported that $55 \%$ of his patients with a ligament rupture had impairment of walking ability after injury compared with $30 \%$ of those without such an injury.

The absence of swelling at the time of the delayed physical examination suggests that there is no ligament rupture (PV neg $=58 \%)$ whereas extensive swelling at this time is indicative of ligament rupture ( $\mathrm{PV}$ pos $=87 \%$ ). Prins (1978), Nilsson (1982) and van der Ent (1984) calculated the positive predictive values to be $70 \%, 63 \%$ and $76 \%$, respectively. The reason for these lower values for swelling may be because these authors performed their physical examination before the fourth day after injury, while in our patients this took place after four days.

Pain on palpation of the anterior talofibular ligament was present in all patients with ligament rupture, confirming the findings of Broström (1965b) and Prins (1978).

No single symptom or test provides complete accuracy of diagnosis. The most reliable single diagnostic feature of a ligament lesion was the presence of a haematoma $(\mathrm{PV}+92 \%)$ or a positive anterior-drawer test $(\mathrm{PV}+90 \%)$. The combination of tenderness at the level of the anterior talofibular ligament, lateral haematoma discoloration and a positive drawer test indicated a ligament lesion in $95 \%$ of cases. A negative drawer test and the absence of discoloration always indicated an intact ligament, as did the absence of pain on palpation at the anterior talofibular ligament.

The interobserver variation proved reliable and the experience of the investigator was of limited importance. Our results and the observations of Stiell et al (1994) suggest that a patient who presents with a painful ankle should be examined according to the Ottawa Ankle Rules to rule out a fracture. Patients without a fracture should then be advised to elevate the leg, apply ice intermittently and limit their walking. A compressive or elastic bandage may be applied. A further physical examination five days later can then rule out a ligament lesion.

The delay in diagnosis, and therefore in definitive treatment, does not impair recovery and healing since the current treatment of choice by functional taping can be started after the swelling has subsided.

No benefits in any form have been received or will be received from a commercial party related directly or indirectly to the subject of this article.

\section{REFERENCES}

Brooks SC, Potter BT, Rainey JB. Treatment for partial tears of the lateral ligament of the ankle: a prospective trial. $\mathrm{Br}$ Med J 1981;282:606-7.

Broström L. Sprained ankles: clinical observations in recent ligament ruptures. Acta Chir Scand 1965;130:560-9.

van der Ent FWC. Lateral ankle ligament injury. Thesis. Rotterdam Elinkwijk, Utrecht 1984.

Kannus P, Renström P. Treatment for acute tears of the lateral ligaments of the ankle: operation, cast or early controlled mobilization. J Bone Joint Surg [Am] 1991;73-A:305-12.

Lindstrand A. Lateral lesions in sprained ankles. Lund, 1976a.

Lindstrand A. New aspect in the diagnosis of lateral ankle sprain. Orthop Clin North Am 1976b;7(1):247-9.

Nilsson S. Sprains of the lateral ankle ligaments: an epidemiological and clinical study with special reference to different forms of conservative treatment. Part I. Epidemiological and clinical considerations. J Oslo City Hosp 1982;32:3-29.

Prins JG. Diagnosis and treatment of injury to the lateral ligament of the ankle: a comparative clinical study. Acta Chir Scand 1978;Suppl 486:3-149.

Stiell IG, McKnight RD, Greenberg GH, et al. Implementation of the Ottawa ankle rules. JAMA 1994;271:827-32. 